\title{
Application of Survey Technology in Geotechnical Engineering Investigation
}

\author{
Xiang $\mathrm{Gao}^{1}$ \\ ${ }^{1}$ Wenzhou Ouyue Land Survey Co., Ltd. Wenzhou, Zhejiang 325000
}

\begin{abstract}
With the continuous development of the construction engineering industry, geotechnical engineering construction has attracted more and more attention from the society. In the construction process of geotechnical engineering, scientific and reasonable engineering investigation is the key to ensuring construction quality and improving engineering safety. Based on this, this article analyzes the application of measurement technology in the process of geotechnical engineering investigation. It is hoped that through this analysis, the application effect of measurement technology in geotechnical engineering survey can be effectively improved, and the quality of geotechnical engineering survey can be improved, so as to promote the overall construction quality and safety of geotechnical engineering.
\end{abstract}

\section{Introduction}

In the construction of geotechnical engineering areas, a key content is to do a good job in geotechnical engineering. Through the corresponding measurement technology, it should carefully survey the actual geology and lithology of the geotechnical engineering construction site, and it should use the obtained survey data as the basis, according to the actual construction needs, it should combine with the actual situation on the site, and it should take scientific measures to carry out construction of geotechnical engineering. Only in this way can the construction quality of the construction project under geotechnical engineering conditions be effectively guaranteed and the safety of the overall construction project can be improved. Therefore, in the specific construction, the construction unit must scientifically apply the measurement technology in the geotechnical engineering survey, so that its technical advantages can be fully utilized to ensure the survey effect.

\section{Comprehensive survey technology analysis in geotechnical engineering investigation}

\subsection{Application principle of comprehensive measurement technology}

In today's geotechnical engineering survey process, comprehensive survey technology is the most advanced and commonly used type of survey technology. The socalled comprehensive measurement technology is to combine two or more measurement technologies to form a comprehensive technical system. On the basis of the characteristics and advantages of single-phase measurement technology, it also helps to compensate each single shortcomings of measurement technology, so as to obtain better measurement results ${ }^{[1]}$. Through the application of this technology, the specific geotechnical conditions and geological condition parameters in the geotechnical engineering measurement area can be obtained more accurately, and the measurement efficiency and measurement accuracy can be comprehensively improved.

\section{Application advantages of comprehensive measurement technology}

Compared with the traditional single measurement technology, the main advantages of applying comprehensive measurement technology to geotechnical engineering survey work are as follows:

Table1. The main application advantages of comprehensive survey technology in geotechnical engineering investigation

\begin{tabular}{|c|c|c|}
\hline $\begin{array}{c}\text { serial } \\
\text { number }\end{array}$ & $\begin{array}{l}\text { application } \\
\text { advantage }\end{array}$ & specific situation \\
\hline 1 & $\begin{array}{l}\text { The equipment is } \\
\text { lighter and smaller }\end{array}$ & $\begin{array}{l}\text { In the application of this kind of } \\
\text { measurement technology, the } \\
\text { instruments and equipment used are } \\
\text { all latest types, which are smaller in } \\
\text { size, lighter in weight, and more } \\
\text { convenient to carry and operate. } \\
\text { Normally, only one or two staff can } \\
\text { complete the entire project of survey } \\
\text { work. }\end{array}$ \\
\hline 2 & $\begin{array}{l}\text { Very high benefit } \\
\text { for the environment }\end{array}$ & $\begin{array}{l}\text { In the process of geotechnical } \\
\text { engineering survey through } \\
\text { comprehensive survey technology, } \\
\text { there is no need to clean up the } \\
\text { vegetation on site, nor will it cause } \\
\text { damage to the ecological } \\
\text { environment. }\end{array}$ \\
\hline
\end{tabular}




\begin{tabular}{|c|c|}
\hline $\begin{array}{c}\text { Very high } \\
\text { measurement } \\
\text { accuracy }\end{array}$ & $\begin{array}{l}\text { Compared with a single form of } \\
\text { measurement } \\
\text { comprehensive technology, } \\
\text { technology can effectively avoid } \\
\text { accuracy fluctuations. With the help } \\
\text { of multiple technical means, repeated } \\
\text { surveys can achieve a large amount of } \\
\text { effective data acquisition, minimize } \\
\text { measurement errors and improve } \\
\text { measurement accuracy. }\end{array}$ \\
\hline
\end{tabular}

\section{Specific application analysis of comprehensive survey technology in geotechnical engineering investigation}

\subsection{Application of shallow seismic reflection wave measurement technology}

Shallow seismic reflection wave measurement technology is a comprehensive measurement technology that uses artificial methods to excite seismic waves to measure the propagation of seismic waves in the medium and judge the geological structure in the measured area. The main application principle of this technology is similar to valley echo. In specific applications, surveyors will excite seismic waves through corresponding instruments and equipment, and then collect information on the propagation speed of seismic wave signals in rock and soil media, and measure the amplitude and frequency of seismic waves. Parameters are used as the basis to judge the specific form of shallow lithology and interface depth in the geotechnical engineering measured area.

At the same time, if the main propagation characteristics of seismic waves are used as the basis, we can divide the shallow seismic reflection wave measurement technology into three types, one is the transmission wave measurement technology; the second is the refraction wave measurement technology; the third is the reflection wave measurement technology. Among the three measurement techniques, the most commonly used shallow seismic wave measurement technique is the reflected wave measurement technique. In the process of geotechnical engineering investigation through this technology, the applied seismic sources cannot bring pollution or damage to the ecological environment, such as electromagnetic pulse generators, piezoelectric transducers, etc. These seismic sources mainly generate piezoelectricity through the release of electric pulses, and then excite the seismic wave ${ }^{[2]}$.

In addition, in the process of selecting seismic sources, the frequency characteristics of seismic signals and their identification should also be fully considered. In this way, the measurement accuracy can be effectively guaranteed, and the measurement task can be completed with quality and quantity.

\subsection{Application of high-density resistivity measurement technology}

The high-density resistivity measurement technology is a survey method in the form of an array. During the survey of geotechnical engineering through this technology, the difference in the conductivity of the rock medium and the soil medium in the measured area of the geotechnical engineering is mainly taken as the basis, a stable current field is manufactured artificially on site. With the help of the current field, the current will be conducted in the ground in the measured area according to a specific distribution law. Next, the surveyor needs to collect the data of each measurement point in the observation section, in order to achieve accurate acquisition of the actual geological conditions and the distribution status of the section in the measured area. In the process of field measurement, the surveyor can directly set up several electrodes at each measurement point in the measured field observation profile. By operating the electrode conversion device and electrical measuring instrument and other equipment, the corresponding data collection can be made. It is completed in a short time with high quality and quantity, and then the collected data is imported into the computer for corresponding processing, and finally the rapid generation of the geoelectrical crosssectional map can be realized.

As far as the technical principle is concerned, this technology and the traditional on-resistivity measurement technology have basically the same principles. The main difference between the two is that the technology has a high-density setting of measurement points in the observation section, so that the geology accuracy of information acquisition of the ground section can be improved. This technology organically combines electrical profile measurement technology, electrical probe technology, and ordinary resistivity measurement technology to give full play to the advantages of these technologies. The following are the specific application advantages of this technology:

Table2. Specific application advantages of high-density resistivity measurement technology

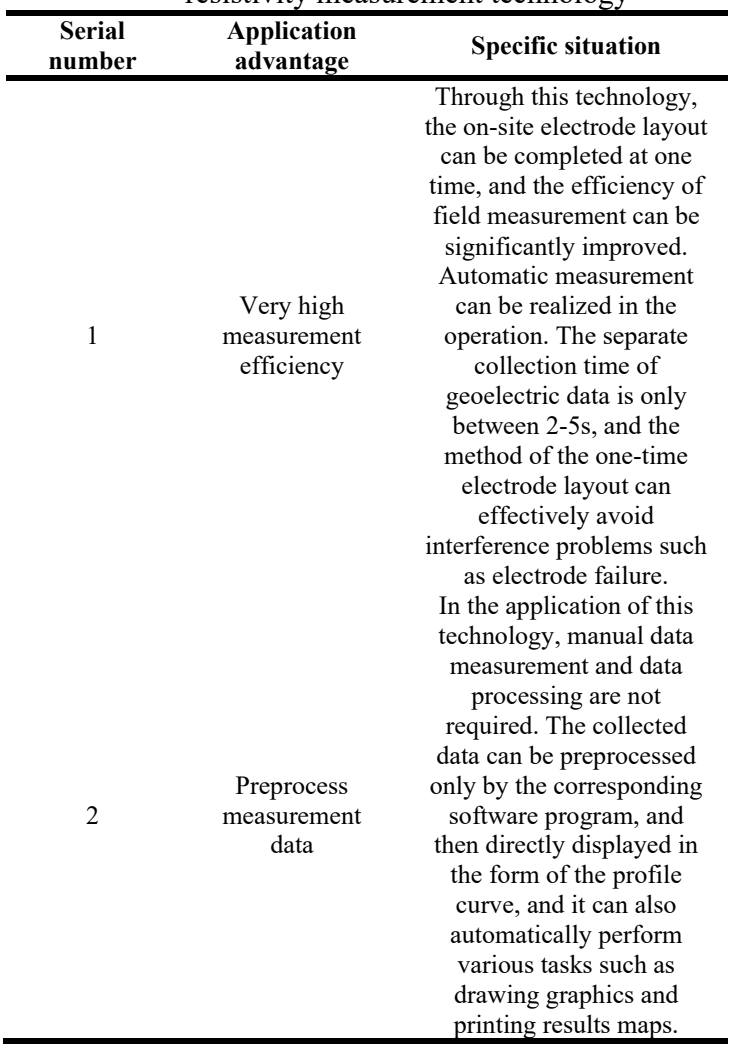




\begin{tabular}{cc}
\hline & $\begin{array}{c}\text { The biggest innovation of } \\
\text { this technology is the array } \\
\text { scanning of the } \\
\text { gery rich }\end{array}$ \\
geotechnical engineering \\
measured area through a \\
information & variety of electrodes, so \\
& that very rich geoelectrical \\
& cross-sectional structure \\
& and geological information \\
& can be obtained. \\
\hline
\end{tabular}

\subsection{Application of geoelectric field lithology measurement technology}

In the process of geotechnical engineering exploration through this technology, the source of the excitation field is electromagnetic waves formed in the solar wind. In specific applications, the surveyor continuously receives electromagnetic waves reflected from various positions in the measured area through the method of point frequency recording, and then comprehensively analyzes parameters such as resistivity and electromagnetic wave amplitude at different positions and depths to achieve this so as to form comprehensive judgment of the characteristics of specific rock formations in the tested area. In the process of geotechnical engineering investigation by this technology, the main application process includes the following aspects: first, import the original data; second, preview the imported data; third, perform the data according to actual needs; fourth, import the corresponding data in the computer and process it; fifth, generate the CYT curve; sixth, re-import the unqualified CYT curve in the computer; seventh, center the CYT curve ; Eighth, to draw the CYT curve; Ninth, to debug the horizontal and vertical ratio according to actual needs; Tenth, to output the CYT expected curve; Eleventh, comprehensively analyze and explain the specific lithology of the tested area according to the output CYT curve $\mathrm{e}^{[3]}$.

In the specific application of this technology, the main equipment is the CYT detector. At present, the most commonly used CYT detector is the CYT-V1 detector. The following is the actual shot of the detector:

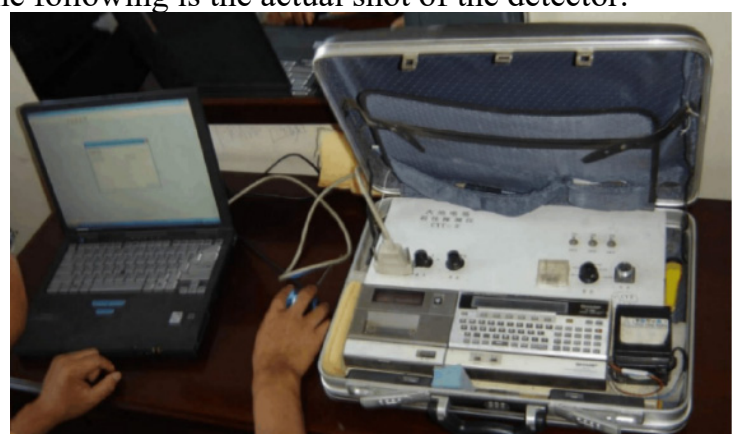

Figure 1. CYT-V1 type detector application real shot diagram In the process of geotechnical engineering geoelectric field lithology measurement by CYT-V1 detector, its main application advantages are shown in the following aspects:

Table3. Main application advantages of CYT-V1 detector in geoelectric field lithology measurement in geotechnical engineering

\begin{tabular}{ccc}
\hline $\begin{array}{c}\text { Serial } \\
\text { number }\end{array}$ & $\begin{array}{c}\text { Application } \\
\text { advantage }\end{array}$ & Specific situation \\
\hline 1 & $\begin{array}{c}\text { Lightweight and } \\
\text { compact }\end{array}$ & $\begin{array}{c}\text { The CYT-V1 detector has a small } \\
\text { volume and a light weight, and is }\end{array}$ \\
\hline
\end{tabular}

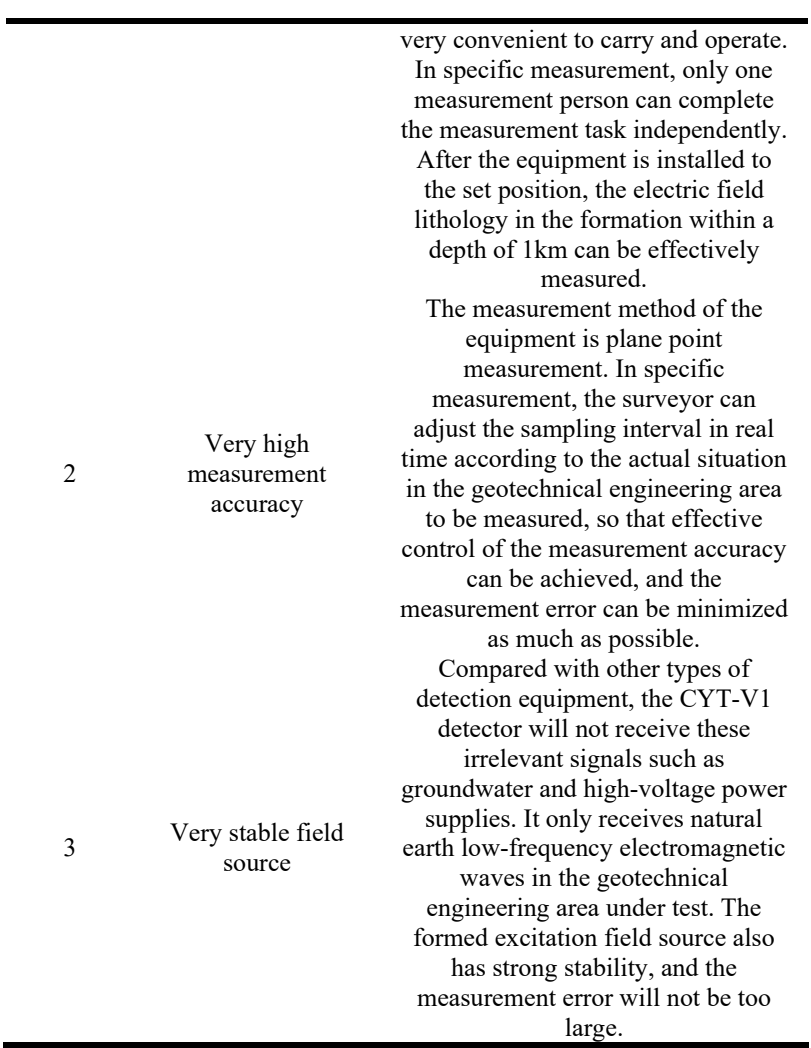

\subsection{Application of multi-transient wave measurement technology}

In the process of geotechnical investigation by multitransient wave measurement technology, the surveyor should set up the sensing device in advance in the stratum structure in the measured area. The seismic source is the transient impact force, and the seismic source will be in the pulse under the action of the load, the ground of the measured area will fluctuate, and the ground wave will be continuously sent to the ground. Next, the surface wave will continue to propagate along the surface of the medium. During the propagation process, the sensor will record the vertical distribution of the wave below. Finally, the surveyor can analyze and process the collected data information accordingly.

In the specific measurement, because the surface waves in different media will have different propagation speeds and propagation characteristics, the geological structure and geotechnical characteristics in the measured area of the geotechnical engineering can be judged according to the specific change law of the dispersion curve. In this way, the survey effect of geotechnical engineering is guaranteed. In addition, the multi-transient surface wave measurement technology can be divided according to two measurement methods, one is the transient measurement method, and the other is the steadystate measurement method. These two measurement methods have different application ranges and operating procedures. It is necessary to make a reasonable choice based on actual needs and combined with the actual situation in the specific applications ${ }^{[4]}$. 


\section{Conclusion}

In summary, geotechnical engineering construction has become a common construction form in today's construction engineering. In the specific construction process, in order to effectively guarantee the quality of the construction, improve the safety of the overall project, and avoid the adverse effects of geotechnical engineering conditions on the construction as much as possible, the construction unit should improve the geotechnical engineering survey, pay attention to it and do a good job in geotechnical engineering surveys through reasonable surveying techniques. In specific surveys, shallow seismic reflection wave measurement technology, high-density resistivity measurement technology, geoelectric field lithology measurement technology, and multi-transient surface wave measurement technology are all commonly used and effective measurement techniques. It needs to choose the corresponding measurement technology reasonably, and do a good job in the reasonable setting and application of the measurement machinery and equipment. In this way, the measurement accuracy can be effectively improved, and the actual survey effect of geotechnical engineering can be guaranteed. This will have a very positive effect on the improvement of the construction quality of construction engineering under the conditions of geotechnical engineering and the development of the construction engineering industry.

\section{Reference}

1. Wang Xinfu. Research on hydrogeological related issues in the process of geotechnical engineering geological survey[J]. Metallurgical Management, 2020(23): 87-88.

2. Zhang Deming. Geotechnical engineering technology innovation method and practice[J]. World Nonferrous Metals, 2020(23):188-189.

3. Zhang Guoyi. The significance of geological analysis in the geotechnical engineering survey of housing construction in Guiyang Pioneer Park[J]. Metallurgy and Materials, 2020(03): 128-129.

4. Chen Jianguo. Discussion on quality control factors in geotechnical engineering geological survey[J]. Jiangxi Building Materials, 2019(09): 62-63. 